\title{
Implementando el coaching educativo en la universidad virtual, una herramienta de desarrollo personal
}

\section{Implementing educative coaching in virtual university, a personal development tool}

\author{
Rosa Domínguez Martín ${ }^{1}$, Ana María Cruz Chust ${ }^{2}$, María Lourdes Ferrando Rodríguez ${ }^{3}$ \\ ${ }^{1}$ Departamento de Educación. Universidad Internacional de Valencia, España (rosa.dominguez@campusviu.es) \\ ${ }^{2}$ Departamento de Educación. Universidad Internacional de Valencia, España (amcruz@ universidadviu.com) \\ ${ }^{3}$ Departamento de Educación. Universidad Internacional de Valencia, España (mlferrando@universidadviu.com)
}

Recibido el 1 de junio de 2018; revisado el 29 de octubre de 2018; aceptado el 29 de octubre de 2018; publicado el 2 de diciembre de 2018

\section{RESUMEN:}

En este artículo se revisa el concepto del coaching entendido como una herramienta de desarrollo personal cuya base es el acompañamiento y cuya finalidad es construir cambios y mejoras encaminados a la consecución de unos objetivos planteados. Si nos centramos en educación, como es el caso, los objetivos están relacionados con el proceso de enseñanza-aprendizaje y los obstáculos serán aquellos relacionados con las dificultades que podemos encontrar en este proceso.

Se revisan, así mismo, experiencias en el entorno educativo universitario que han arrojado resultados positivos en el rendimiento de los estudiantes, en su competencia de autogestión, en su motivación hacia los estudios y en su autonomía para identificar y seleccionar estrategias para avanzar en sus objetivos académicos y profesionales.

En esta revisión, queda patente que es en entornos de enseñanza-aprendizaje virtuales donde los estudiantes necesitan un apoyo más transversal, directo y personal que el prestado por el profesorado en cada una de sus materias. Esto puede deberse a que el perfil del estudiante de esta modalidad, adulto trabajador, necesita un acompañamiento más intenso para poder afrontar con éxito la incertidumbre del entorno, la soledad, la conciliación de sus diferentes responsabilidades, la gestión del tiempo y la actualización de sus competencias académicas y digitales.

En este sentido, la Universidad Internacional de Valencia se suma, con un programa piloto de coaching para estudiantes de primer curso de grado, a una de las innovaciones más directamente relacionadas con el acompañamiento al alumnado en entornos virtuales de aprendizaje. Innovación que, a priori, guarda una relación directa con la mejora del rendimiento de los estudiantes, el desarrollo de competencias, la motivación y, por tanto, la persistencia en los estudios.

Como resultados más destacados de la experiencia piloto, cabe señalar que participaron 64 estudiantes, y un $83,3 \%$ consideró que el programa mejoró tanto sus competencias académicas como sus habilidades personales. Asimismo, el $91 \%$ se sintió satisfecho con el programa y lo recomendaría a sus pares en futuras ediciones.

PALABRAS CLAVE: COACHING, ENTORNOS VIRTUALES, RETENCIÓN ESTUDIANTIL, PERSISTENCIA ACADÉMICA, DESARROLLO PERSONAL Y PROFESIONAL, MOTIVACIÓN.

\section{ABSTRACT:}

In this paper the concept of coaching understood is reviewed as a personal development tool whose base 
is the accompaniment and whose purpose is to build changes and improvements aimed at achieving certain objectives. If we focus on education, the objectives will be related to the teaching-learning process and the obstacles will be those related to the difficulties we can find in this process.

Some experiences are also reviewed in the university area that have showed positive results in the student's competence of self-management, their motivation towards studies and their autonomy to identify and select strategies to achieve their objectives, academics and professionals.

In this review it is clear that it is in virtual teachinglearning environments where students need a more transversal, direct and personal support than the one provided by the teaching staff in each of their subjects.

This may be due to the fact that the student profile of this modality, adult worker, needs a more intense accompaniment to successfully face the uncertainty of the environment, loneliness, the reconciliation of their different responsibilities, the time management and updating of their academic and digital competences.

In this sense, the Valencia International University joins, with its experience of coaching among its first-year students, one of the innovations most directly related to the generation of spaces for guidance, guidance and support for students in virtual environments. Innovation that, at first, has a direct relationship with the improvement of student performance, the development of skills, motivation and, therefore, the persistence of studies.

As the most outstanding results of the pilot experience, it should be noted that 64 students have participated and $83.3 \%$ of them considered that the program improved their academic and their personal skills. Likewise, $91 \%$ were satisfied with the program and would recommend it to their peers in future editions.

KEYWORDS: COACHING, VIRTUAL ENVIRONMENTS, STUDENT ATTRITION, ACADEMIC PERSISTENCE, INDIVIDUAL AND PROFESSIONAL DEVELOPMENT, STUDENT MOTIVATION.

\section{COACHING EDUCATIVO, CONCEPTO E IMPORTANCIA}

\section{1 ¿Qué es el coaching educativo?}

El término "coaching" ha llegado de unos años a la actualidad al español. Su homónimo podría ser acompañamiento.
La palabra coach, del inglés, significa literalmente "carruaje" y, por asociación, "transporte". En la línea de Muradep (2012) etimológicamente, el coach es aquella persona que conduce a otra para que llegue a otro lugar; por ello, un entrenador conduce en el mundo deportivo al desarrollo del potencial de un deportista o un equipo. El castellano lo ha adoptado porque, en principio, no hay un sinónimo que describa con tanta precisión a qué nos estamos refiriendo cuando hablamos de un coach.

Hacer coaching, por tanto, es acompañar a otra persona para conducirla al éxito, para potenciar su desarrollo y apoyarla en ese proceso.

Como se ha comentado, originalmente el término coach significaba carruaje, pero los estudiantes de la Universidad de Oxford comenzaron a darle, de forma coloquial, otro sentido al llamar así a la persona que les ayudaba a superar los exámenes tras identificar sus puntos débiles. Años más tarde los entrenadores que ayudaban a los deportistas a superar sus limitaciones y alcanzar sus objetivos fueron denominados "coaches" (De Andrés, 2014).

Nos encontramos ante un concepto aplicable a muchas áreas, incluso inconexas entre ellas. Hoy en día encontramos este concepto relacionado con el mundo empresarial, deportivo, educativo, económico, psicológico, familiar y demás cuyo nexo esencial de unión es el acompañamiento externo.

El coaching personal pretende guiar sobre las relaciones con los demás, sobre el desempeño profesional, incluso sobre la gestión de la propia economía. En definitiva, busca el equilibrio de la persona que lo solicita para que consiga alcanzar las metas que se propone de forma exitosa y eficaz.

En el ámbito personal, también encontramos la especialidad del coaching ejecutivo, que se centra en guiar a aquellas personas que ocupan posiciones de responsabilidad en la toma decisiones empresariales y en la gestión de personas.

Por otro lado, en el mundo propiamente empresarial, encontramos el coaching organizacional que pretende favorecer el desarrollo de la organización como grupo, sumando el compromiso de cada individuo para el éxito común y construyendo cultura a partir de la relación entre las personas que intervienen y el apoyo entre ellos.

Estudios como el de López-Pérez y VallsBallesteros (2015) se centran en el campo educativo señalando que el coaching es una forma de acompañamiento cuyo objetivo es el aprendizaje, desarrollo y crecimiento de individuos o grupos de una forma personalizada. 
El coaching ofrece una vía de observación para pensar, reflexionar y profundizar en los retos, de forma que se genere un cambio que provoque el aprendizaje. Este cambio, suele vincularse con los factores emocionales y relacionales; por ello, el coach adquiere gran valor como agente de acompañamiento.

Ramírez-Echeverri (2014) plantea el coaching como una técnica de asesoramiento con la que transformar, desde competencias laborales hasta personales y emocionales, incluso como cambio en equipos de trabajo.

Otros trabajos como el de Bou (2009) definen el coaching como aquel proceso de aprendizaje que es sistemático y se centra en la situación presente para provocar el cambio, facilitando recursos $\mathrm{y}$ herramientas de trabajo específicos.

Estamos tratando, por tanto, un concepto cuya base es el acompañamiento y cuya finalidad es construir cambios y mejoras encaminados a la consecución de unos objetivos planteados. Si nos centramos en educación, los objetivos estarán relacionados con el proceso de enseñanzaaprendizaje y los obstáculos serán aquellos relacionados con las dificultades que podemos encontrar en este proceso.

Entonces ¿qué significa ser coach? ¿cuáles son las funciones de un coach? ¿hasta dónde podemos ser guiados por nuestro coach?

Para responder a estos interrogantes y profundizar en la temática estableceremos los siete elementos constitutivos del coaching, sea cual fuere la especialidad del mismo (Bécart y Ramírez, 2016):

a) Ser coach es ser acompañante o entrenador de alguien.

b) El coaching se caracteriza por contar con técnicas y herramientas profesionales.

c) Siempre se dirige hacia la mejora, buscando alcanzar los objetivos marcados y superar los obstáculos, físicos o psicológicos.

d) Es quien recibe el coaching quien debe decidir qué necesita mejorar y sobre qué profundizar- más tarde, el coach descubrirá si hay más puntos sobre los que trabajar-.

e) El coaching implica una intención de mejora y por tanto, conllevará un cambio, siempre dirigido en positivo.

f) Su fin último es desarrollar competencias de forma efectiva y eficaz.

g) Se trabaja de forma holística e integral para que el beneficio repercuta en la persona como totalidad.

\section{2 ¿Por qué coaching educativo en los entornos virtuales de aprendizaje (EVA)?}

En este trabajo partimos de una realidad concreta, la universidad virtual.

Ya hace mucho de aquella universidad en la que el docente obligaba a los estudiantes a ir presencialmente al aula, siendo las clases magistrales la base del conocimiento. La accesibilidad a la información a través de la red y la tendencia a universalizar el acceso a este nivel educativo, han propiciado el cambio de paradigma gracias a las oportunidades que ofrece. Actualmente, es más crítico desarrollar la competencia de gestión de la información que la adquisición del conocimiento en sí misma, ya que esta puede encontrarse en multitud de portales de Internet al alcance de un "clic".

Nos encontramos, así, ante una universidad en la que se puede aprender desde cualquier sitio y en cualquier momento. De esta forma, se potencia que personas con horarios complicados o responsabilidades laborales y/o familiares, cuyo tiempo es muy escaso, puedan formarse para ampliar sus conocimientos o bien cumplir el sueño de tener una titulación que, por motivos diversos, no han podido conseguir con anterioridad.

El campus virtual es ahora el lugar de encuentro y comunicación en el que se convive y, junto con los correos electrónicos, la información viaja de una parte a otra del planeta. Desde el punto de vista pedagógico, las limitaciones son ínfimas en comparación con las oportunidades de esta metodología en la que, simplemente, hay que tener una conexión a Internet.

¿Cuál es la función entonces de un coach en los EVA? Es bien sabido que los seres humanos somos animales sociales, necesitamos de nuestros homónimos y de la relación con ellos para vivir. La educación online potencia la relación entre personas que están a kilómetros de distancia, pero puede hacer que esta sea meramente académica y formal, ya que inevitablemente la riqueza del lenguaje no verbal (gestos, miradas y posturas) queda excluida, o bien, es más difícil de trasladar.

En el entorno universitario virtual, nos encontramos con un alumnado que, en su mayoría, es adulto y tiene multitud de compromisos (sean familiares, laborales o de otro ámbito), en algunos casos con estudios previos, ya establecidos profesionalmente pero que quieren formarse, mejorar, cumplir un sueño postergado y quieren hacerlo de la mejor forma posible. 
El camino de estos estudiantes se inicia con altas expectativas e ilusiones que, en un primer momento, pasan por afrontar con éxito un entorno de cierta complejidad tecnológica, para continuar con una metodología diferente a la que vivieron en otras etapas de su vida académica, con un lenguaje fácil a primera vista, pero complejo en su fondo, con estrategias de estudio un tanto oxidadas y respondiendo a unos plazos que, necesariamente, tienen que encajar con las diferentes facetas de sus vidas.

En este punto, altamente sensible de inicio de la experiencia universitaria en un EVA, adquiere especial importancia la figura del coach. En la universidad virtual, un coach es un profesional que no es docente de las personas que guía. Es un agente externo que acompaña y da el apoyo necesario a los estudiantes en estos primeros momentos de incorporación a la universidad. El coach tiene relación transversal con el claustro y otros departamentos para poder responder a las demandas del estudiante y que las relaciones internas puedan ser más fluidas.

Algunas personas solicitan de su coach, alguien que entienda el mundo de la universidad en el que están inmersos, alguien a quien plantear las dificultades de volver a poner a punto las habilidades de estudio y con quien compartir las preocupaciones ante las competencias digitales por adquirir.

Otras, simplemente necesitan una persona con la que compartir satisfacciones, éxitos en las calificaciones, esfuerzos y demás. No podemos olvidar que la relación entre los compañeros no se da necesariamente, aunque se conozcan por estar juntos en las mismas asignaturas, ya que se conectan a las aulas virtuales en horarios y lugares totalmente diferentes. Por tanto, el compañerismo entre estudiantes puede darse o no, por lo que, para algunas personas, el coach se convierte en esa persona que entiende el mundo en el que se está actuando y con el que se puede compartir un lenguaje común.

También hay personas que necesitan que les guíen en la organización del tiempo y del material ya que, en la educación virtual, se requiere de autonomía, capacidad de autogestión y de habilidades organizativas para encontrar los momentos idóneos en los que estudiar y realizar las tareas, sin descuidar el resto de responsabilidades personales y profesionales. El coach, ofrece claves para que tanto el tiempo invertido, como el esfuerzo y la implicación realizados, ofrezcan sus frutos más adelante.
Superar estas primeras semanas de incertidumbre y ambigüedad en un entorno virtual y llevar al día las asignaturas es todo un reto para los estudiantes adultos. El nivel de ansiedad es elevado y, a veces, bloqueante, pero cierto es que disminuye cuando se verbaliza y se comparte con el coach. Esto hace que el ambiente de estudio creado favorezca el proceso aprendizaje, y con él los primeros logros y éxitos académicos.

Si entendemos esta afirmación como certera, parece evidente que, en la formación online, más que importante es esencial este acompañamiento, este apoyo, ya que una de las principales carencias es el afecto y conexión que se crean entre las personas al relacionarse de forma presencial.

\section{INICIATIVAS DE COACHING EDUCATIVO CON RESULTADOS EXITOSOS}

\subsection{Implicaciones del coaching en el acompañamiento a profesores $y$ estudiantes}

Las posibilidades e implicaciones del coaching en áreas relacionadas con el cambio, la gestión de equipos y la resolución creativa de conflictos ha ido aumentando el número de investigaciones y publicaciones en ámbitos educativos, tanto a nivel de innovación, gestión del talento y acompañamiento al equipo directivo en el desarrollo de su rol en los centros educativos, como a nivel de las propias aulas, indagando en medidas de acompañamiento a profesorado y a alumnado en la mejora de los resultados académicos.

Son cada vez más los estudios que demuestran que, cuando los equipos docentes utilizan el coaching como metodología, aumenta considerablemente la sensación de éxito de los estudiantes y las mejoras en los resultados académicos de los segundos son evidenciables (Ruiz et al., 2014; Sánchez y Boronat, 2014).

Así, esta poderosa herramienta de cambio orientada hacia el éxito, relativamente reciente y en boga en el mundo empresarial (Bou, 2013) es cada vez más valorada desde la comunidad educativa. Como se verá en el apartado siguiente, en base a las evidencias empíricas aportadas por investigaciones recientes, el coaching viene siendo utilizado como estrategia, e incluso, como modelo para la mejora sustancial de los procesos educativos.

En España, una de las primeras publicaciones sobre coaching aplicado a la educación aparece en la Revista de Orientación y Psicopedagogía (Bisquerra, 2008) como técnica a considerar en 
orientación (Sánchez y Boronat, 2014). En la actualidad, encontramos publicaciones que incluyen aspectos, conceptos y aplicaciones con resultados exitosos relacionados con el coaching. En general, estos estudios vienen asociados a iniciativas llevadas a cabo en centros educativos y a la formación del profesorado de distintos niveles educativos en técnicas y herramientas relacionadas con el éxito escolar.

A continuación, se exponen algunas experiencias en entornos educativos, donde el coaching ha formado parte del éxito académico de los procesos de enseñanza-aprendizaje implicados. Estos resultados muestran que la apuesta, y la puesta en marcha de esta importante herramienta, puede ser una parte relevante de la metodología de trabajo del profesorado para acompañar a los estudiantes en un proceso de aprendizaje que tenga como base las preguntas y las intervenciones surgidas en el seno del aula.

A nivel de universidad no abunda la difusión de experiencias sobre procesos de coaching vinculados al acompañamiento de estudiantes universitarios. Y eso, a pesar de que en el argot universitario inglés del siglo XVIII ya se hacía alusión a un tipo particular de profesor que ayudaba a un estudiante a prepararse, rápida y eficientemente, para los exámenes (Sánchez y Boronat, 2014).

\subsection{Experiencias de éxito vinculadas al coaching en entornos universitarios}

Ruiz et al. (2014) refieren brevemente una experiencia de coaching educativo en el grado de Psicología, concretamente en la asignatura optativa de Psicología de los Recursos Humanos, con treinta alumnos, durante ocho semanas de clase y una duración de treinta horas.

El equipo de investigación asocia a la metodología de coaching los resultados positivos. Así, algunas de las actuaciones que destacan son: los procesos vinculados al rol del profesorado en el aula, quienes promueven la reunión de los estudiantes en parejas de trabajo y les dan feedback sobre la sesión, la supervisión de las áreas de mejora, la formación en metodologías como GROW y Johari Windows, y la facilitación de espacios para la evaluación del plan de acción de cada discente.

En la metodología seguida, las sesiones de coaching se realizaban durante una hora por parejas (treinta minutos por coacheelcoach). Cada semana y libremente, cada alumno escogía su coach-partner del aula.

A nivel de objetivos, cada estudiante/coachee debía auto observarse, realizando un proceso de introspección, detectando diez puntos fuertes y diez puntos de mejora. Por otra parte, debía contactar con dos observadores externos (padres, amigos, pareja, etc.) para que, de forma independiente, le indicara diez puntos fuertes y diez puntos de mejora. A partir de estos datos, el discente hacía un análisis de los mismos, eligiendo los diez más importantes para trabajar en un plan de acción personalizado, compartido con el coach-partner del aula, que supervisaba los avances o retrocesos de los compromisos adquiridos en el plan de acción

Vivir en "directo" un proceso de coaching en el aula en los dos roles, el de coach y el de coachee, entregando por escrito el plan de acción y los compromisos adquiridos, formó parte de la experiencia.

Otro ejemplo de iniciativas de coaching, como tendencia educativa, viene de la mano de procesos de mentoring en Latinoamérica. Así, desde el Observatorio de Innovación Educativa del Instituto Tecnológico de Monterrey (2017), se han impulsado una serie de iniciativas vinculadas a proyectos personales de los estudiantes y a emprendimientos institucionales para lograr los objetivos de cada uno de forma articulada. Aquí, en este contexto, el mentor resulta una pieza clave para llevar a cabo el seguimiento estratégico de las actividades en las que se involucran los estudiantes, otorgándoles la corresponsabilidad de la consecución de las metas planteadas. En estas experiencias educativas el mentor motiva, asesora, guía y califica; también contribuye a la vinculación profesional de los alumnos con agentes externos a la universidad y propicia el desarrollo de redes que le permiten integrarse al entorno laboral.

Algunas otras experiencias en el contexto de la enseñanza universitaria, vinculadas al coaching, como técnica metodológica que favorece el desarrollo del potencial humano y ayuda a los estudiantes a progresar, vienen propuestas por Sánchez y Boronat (2014). La experiencia se desarrolló durante el curso 2010-2011 con 142 alumnos de tercer curso, de las especialidades de maestro de Educación Infantil y Educación Física en un centro de formación del profesorado, con importante trayectoria en proyectos de investigación, orientados a la innovación y calidad docente: la Escuela Universitaria de Educación de Palencia.

Las investigadoras y docentes apuestan por la creación del conocimiento por parte del alumnado (autoaprendizaje), y por la consideración de la figura del docente como guía del proceso de aprendizaje y facilitador del conocimiento; todo ello, en línea con la filosofía que subyace en el proceso de Bolonia, de 
corte constructivista en el que se reivindica el poder de los estudiantes para aprender.

Para el desarrollo del estudio piloto en la formación de los futuros docentes de aulas universitarias, las autoras se apoyan en los modelos procesuales de Bayón et al. (2006) y de Carril (2008), dada la proyección que puede tener en el ámbito educativo universitario.

Así, las educadoras, toman como punto de partida los principios que constituyen la esencia del proceso de coaching, y que implican la toma de consciencia del coachee para que sean conscientes de sí mismos, siendo capaces de salir de la zona de confort y marcando metas motivadoras que les permitan dar pequeños y continuos pasos siguiendo por un lado su intuición, sin abandonar el ser dueños de su tiempo, reflexionado sobre estrategias para avanzar en los objetivos trazados.

Este proyecto piloto, con claros enfoques subyacentes de desarrollo de competencias, implicó un tratamiento metodológico particular, vinculado a la enseñanza y al desarrollo de habilidades complejas. Su aplicación en la docencia universitaria, muy asociada a la tutoría, implicó un compromiso bidireccional entre el profesorado/coach y el alumnado de las especialidades de Magisterio de Educación Infantil (64 alumnos) y Educación Física (78 alumnos).

El modelo es una clara apuesta para que los jóvenes lleguen a ser inconscientemente competentes en la resolución de problemas, dado que, como refieren las docentes y afirma Bayon (2006), no hay una solución preestablecida en los libros.

La aplicación de este modelo piloto de coaching, liderado por las dos profesoras implicadas, resultó muy positivo, dado que los estudiantes pudieron desarrollar y, al mismo tiempo, autoevaluar sus propias competencias.

El abandono universitario es un problema que preocupa en todas las instituciones de educación superior. Por ello, muchas universidades han promovido investigaciones que analicen las causas e innovaciones que desarrollan programas de retención de estudiantes. Por eso cerramos este apartado comentando dos experiencias relacionadas con el impacto del coaching en la retención del alumnado universitario.

La experiencia de coaching de la Universidad de Stanford, desarrollada por los profesores Bettinger y Baker (2011), se ha llevado a cabo con estudiantes de primer curso y ha consistido en proporcionarles acompañamiento en su primera experiencia universitaria con el objetivo de aumentar su retención y compromiso con los estudios que estaban cursando. La evaluación del programa arrojó un resultado de 5 puntos porcentuales más de probabilidad de continuar en la universidad para los estudiantes que siguieron el programa de coaching, lo que representó una reducción del 12 al 9\% de abandono estudiantil. Además de esto, se encontró que el efecto del coaching no desaparecía en cursos superiores. Así, los estudiantes que se habían beneficiado del coaching en el primer año tenían más de probabilidades de continuar con sus estudios después de 18 meses y 24 meses.

En el contexto español, la Universidad Politécnica de Madrid (UPM) ha sido una de las instituciones que más ha investigado e innovado para mejorar los índices de permanencia y reducir la tasa de abandono entre sus estudiantes de Ingeniería. Así lo reflejan en su programa de Inteligencia Emocional y coaching en la Universidad (Casado et al., 2012) y su Protocolo de calidad para la tasa de permanencia a un año (Gonzalo at al., 2013). En ambas experiencias los resultados hablan de los beneficios indiscutibles del acompañamiento a los estudiantes, tanto en la adquisición de ciertas competencias transversales como en la incidencia positiva en el mejor aprovechamiento de su potencial intelectual. Ambos aspectos resultan determinantes para el desarrollo integral de los jóvenes ingenieros y su vinculación a los estudios.

\section{EL PROGRAMA "CLAS" DE LA UNIVERSIDAD INTERNACIONAL DE VALENCIA}

Las grandes posibilidades que se derivan de la puesta en marcha de diferentes procesos de coaching y mentoring educativo, nos hacen ratificar la importancia de la reflexión de los estudiantes durante sus respectivos procesos de aprendizaje y los espacios que podemos generar como docentes, guiando, ayudando y acompañando en estos procesos.

En la Universidad Internacional de Valencia VIU- se ha desarrollado una experiencia piloto en el curso 2017-18, a lo largo de sus dos cuatrimestres, vinculada al acompañamiento del alumnado, entendido como el seguimiento académico que este recibe a lo largo de su pupil learning journey.

La finalidad de incorporar esta innovación educativa en la experiencia del estudiante fue doble. Por una parte, se incorporó una acción que individualizara el proceso de enseñanza-aprendizaje virtual y que contribuyera a la mejora del rendimiento académico y el desarrollo de 
competencias. Por otra, se esperaba que los resultados de esta acción ayudasen a mejorar las tasas de permanencia y graduación, así como aumentar la satisfacción de los estudiantes con la institución.

Partiendo de este planteamiento, CLAS, acrónimo de Coaching for Learning and Academic Success, se articuló como programa voluntario de acompañamiento personalizado al estudiante donde la figura del coach pedagógico cobró protagonismo para el primer curso de los programas de grado que forman parte del proyecto piloto.

Bajo la nomenclatura de CLAS se vertebró la acción de un especialista del coaching cuyo perfil profesional se enmarcó en el área de la Pedagogía o afines, ejerciendo roles como el de asesor, educador, consejero, guía, líder, animador, confidente, etc., siendo su misión fundamental convertirse en un acompañante del estudiante durante su primer año de vida como comunidad VIU (primer curso de grado).

La metodología seguida en el Programa se asoció a los siguientes hitos:

a) La realización de una entrevista inicial con el estudiante que permitiese hacer un status del punto de su partida en relación a variables como el número de asignaturas matriculadas, el tiempo que disponía, su perfil de acceso y una primera prospección de sus competencias académicas y digitales frente al proceso de aprendizaje virtual. Así, el primer contacto de los coachees con el coach, fue fundamental en la primera sesión. La entrevista en profundidad ayudó al diseño de un plan de acompañamiento personalizado. Este plan estuvo centrado en las necesidades e intereses vinculados, por una parte, al desarrollo de competencias académicas, y por otro, al desarrollo de competencias personales que promoviesen y facilitasen experiencias de éxito relacionadas con el aprendizaje activo.

El sondeo de habilidades sociales para afrontar tareas en equipo, la gestión que se hacía del tiempo para afrontar las actividades, así como las estrategias cognitivas que utilizaba el alumnado para afrontar sus estudios universitarios en modalidad online, fueron elementos de gran interés para focalizar el acompañamiento.

b) La elaboración conjunta con el estudiante/coachee de un de plan de trabajo individualizado acorde a las necesidades e intereses. c) La revisión de ese plan y la orientación pedagógica y personal hacia objetivos para la superación del portafolio de cada materia, para el aprendizaje, para el desarrollo de la confianza y el propio potencial.

d) La entrevista de cierre, donde se trabajó el fomento de la motivación hacia el Programa, la continuación de los estudios y el sentido de pertenencia a la Universidad.

Dado que el acompañamiento que precisó cada estudiante pudo ser diverso en su naturaleza y frecuencia según las necesidades individuales, se realizó el servicio a través de dos vías fundamentales: videoconferencia y correo electrónico.

Finalmente hay que indicar que, al tratarse de una experiencia piloto, seguimos en fase de validación, valorando desde los distintos roles implicados, la puesta en práctica de la misma para proponer las mejoras que puedan ser pertinentes y que nos permitan en otra publicación, compartir más resultados y conclusiones con la comunidad educativa.

\section{RESULTADOS DE LA EXPERIENCIA PILOTO DEL PROGRAMA "CLAS"}

Hasta el momento los datos recogidos corresponden a una sola edición del Programa CLAS, -octubre 2017-2018-.

En ella, el alumnado que fue susceptible de beneficiarse del Programa se elevó a 328, de los cuales asistieron a la sesión informativa 220 $(67,1 \%)$. Cabe destacar la voluntariedad para inscribirse en el Programa, aspecto que se consideró prioritario al tener como objetivo ofrecer ayuda al alumnado que lo necesitara o demandara el acompañamiento.

Precisamente esta característica de voluntariedad del Programa produjo dos efectos cualitativos en la primera parte de la ejecución del mismo:

1) alejó a algunos estudiantes, que entendieron la participación en el Programa como más carga de trabajo y

2) desvió el foco de atención de los estudiantes abrumados por la novedad y la falta de capacidad para organizarse, que constituían, precisamente, el público objetivo.

Como se ha comentado, la asistencia de más de la mitad de los alumnos convocados $(67,1 \%)$ indicó un interés inicial, pese a que, finalmente, el porcentaje de alumnos que se inscribió en el Programa CLAS se redujo a 64 , un $29,6 \%$. 
Los datos de interacción en la fase central del Programa, en el cuatrimestre de referencia, arrojaron una media de 8 intercambios a través de la comunicación mediante correo electrónico entre estudiantes y coaches y de 2 tutorías individuales por estudiante.

Una vez finalizada la experiencia piloto, se pasó a los discentes una encuesta de satisfacción, con la que verificar los aspectos positivos a potenciar y mejorar aquellos en los que los estudiantes hubieran percibido carencias. La recogida de información se realizó través de una encuesta con una escala tipo Likert donde el alumno se tenía que posicionar entre el 1 "nada de acuerdo" y el 5 "totalmente de acuerdo" en los diferentes ítems.

A partir de las encuestas, podemos extraer los siguientes resultados:

- El $81 \%$ estuvo de acuerdo o totalmente de acuerdo en que los objetivos del Programa estuvieron claros en todo momento, mostrándose el $90 \%$ de acuerdo en que los recursos utilizados fueron de utilidad.

- $\quad$ El 68\% de los estudiantes respondieron estar de acuerdo o totalmente de acuerdo en que el tiempo de acompañamiento a lo largo del curso fue el adecuado.

- Para el 84\% el acompañamiento a través de videoconferencias era bastante adecuado, siendo el $91 \%$ los que prefieren que éste se lleve a cabo a través del correo electrónico.

Se consideró relevante conocer la percepción de los estudiantes sobre el impacto del Programa en la mejora de sus competencias académicas y habilidades personales. A este respecto el $83,3 \%$ consideró que el Programa efectivamente ha mejorado ambos aspectos.

Por último, para tener una visión holística del feedback ofrecido, el $91 \%$ del alumnado manifestó sentirse satisfecho con el Programa, añadiendo que lo recomendarían a sus compañeros en futuras ediciones.

\section{CONCLUSIONES}

A menudo, en la educación universitaria damos por supuesto que los estudiantes saben cómo afrontar los retos académicos. Suponemos que saben cómo estudiar, cómo priorizar, cómo planificar y cómo tomar decisiones solamente porque son adultos $\mathrm{y}$ porque así lo están haciendo en otras esferas de su vida. Sin embargo, dado lo que sabemos sobre las tasas de abandono en la universidad, y sobre todo en la modalidad virtual, esta es una suposición que debería cuestionarse.

El coaching es una herramienta de acompañamiento orientada a la obtención de resultados que ha demostrado su utilidad, tanto en el ámbito profesional, como en el ámbito educativo y académico. Entre sus objetivos puede estar conducir al estudiante a clarificar un problema o dificultad, ayudarle a cuestionar sus creencias y prácticas académicas concretas, permitirle identificar los obstáculos que frenan el desarrollo de sus competencias, descubrir recursos alternativos en los que puede apoyarse, aplicar soluciones personales eficaces trasladadas de otros entornos o esferas de su vida que le permitan alcanzar eficazmente sus objetivos.

A esto se añade, además, que los efectos del coaching y su prevalencia en la motivación de los estudiantes a continuar los estudios, hace que el abandono disminuya. Aspecto sumamente interesante para las universidades con modelos educativos a distancia o virtuales, ya que hace aumentar sus tasas de graduación.

En conclusión, los resultados de las experiencias descritas en este artículo y las que se están poniendo en marcha recientemente, como en la Universidad Internacional de Valencia, sugieren que el coaching educativo es una forma eficiente y eficaz de ayudar a los estudiantes para que tengan éxito en sus estudios universitarios.

Aunque todo apunta en la dirección del efecto positivo del coaching, claramente se necesitan estudios experimentales que utilicen la asignación aleatoria de estudiantes para evaluar realmente los efectos del coaching, así como en qué población de estudiantes es más efectivo. También sería interesante saber si hay características específicas de los coach educativos que aumentan su eficacia. E, incluso, conocer los tipos específicos de servicios de coaching y las acciones específicas de los coaches que son más efectivas para motivar y acompañar a los estudiantes.

\section{REFERENCIAS}

Bayón, F., Cubeiro, J.C., Romo, M., y Sainz, J.A. (2006). Coaching realmente. Madrid: Pearson Prentice Hall.

Bécart, A. (2015). Impacto del coaching en el desarrollo de competencias para la vida. Un estudio de caso en el caribe colombiano (Tesis doctoral). Universidad Pablo de Olavide, Sevilla, España.

Bécart, A, y Ramírez Garrido J, (2016) Fundamentos del coaching educativo: caracterización, aplicaciones y beneficios desde los cuatro pilares del saber. Plumilla Educativa Instituto Pedagógico pp. 344-362. 
Bettinger, E., y Baker, R. (2011). The effects of student coaching in college: An evaluation of a randomized experiment in student mentoring (No. w16881). National Bureau of Economic Research.

Bisquerra, R. (2008). Modelos de orientación e intervención psicopedagógica (No. Sirsi) i9788471978752).

Bou, J. F. (2009). Coaching para docentes: el desarrollo de habilidades en el aula. Alicante: Club Universitario.

Bou, J. F. (2013). Coaching educativo. LID Editorial.

Carrera, L. y Luz, J. (2008). El coaching como estrategia para la formación de competencias profesionales. Escuela de Administración de Negocios, 63, 127-137.

Carril, J. (2008). Zen coaching: un nuevo método que funde la cultura oriental y occidental para potenciar al máximo tu vida profesional y personal. Madrid: Díaz de Santos

Casado, M. L., Sánchez, M. C., López, D., Scheele, S. y Bravo, A. (2012). Inteligencia emocional y coaching en la universidad: consolidación de un proyecto de innovación educativa. En VII Jornadas de Mentoring y Coaching. JIMCUE 2012, Madrid-España. pp. 1-13.

De Andrés, A. (7 de enero de 2014). ¿En qué consiste el coaching educativo? [Entrada de un blog]. Recuperado de: http://blog.smconectados.com/2014/01/07/en-queconsiste-el-coaching-educativo/

Gonzalo, A.I., García, A., Blanco, J., Casaravilla, A., Castejon, Á., Mahillo, Á, y Malinga, M. (2013). Protocolo de calidad para la tasa de permanencia a un año en la Universidad Politécnica de Madrid. En III Conferencia Latinoamericana sobre el Abandono de la Educación Superior (III-CLABES). México. pp. 985-995.

Lárez H., J. (2008). El coaching educativo como estrategia para potenciar el éxito durante la etapa de desarrollo y culminación del trabajo especial de grado. Sapiens. Revista Universitaria de Investigación, 9(2), 219-234.

López-Pérez, C., y Valls-Ballesteros, C. (2015). Coaching educativo. Las emociones al servicio del aprendizaje. España, Madrid: Ediciones SM.

Muradep, L. (2012). Coaching para la transformación personal. Ediciones Granica SA.

Observatorio de Innovación Educativa. Instituto Tecnológico de Monterrey (2017) Mentoring. Reportes Edu Trends.

Ramírez-Echeverri, L.A. (2014). El coaching desafio para orientar equipos de trabajo (Trabajo Fin de Grado). Universidad Militar Nueva Granada, Bogotá, Colombia

Ravier, L. (2005). Arte y ciencia del coaching: su historia, filosofía y esencia. Buenos Aires: Dunken.

Ruiz Callejón, E. (2015) ¿Del 'coaching' al neurocoaching? Algunas reflexiones críticas sobre el coaching educativo en educación superior en la era de la neurocultura. Ensayos de Filosofía, 1. Recuperado de http://www.ensayos-filosofia.es/archivos/articulo/delcoaching-al-neurocoaching-algunas-reflexiones-criticassobre-el-coaching-educativo-en-educacion-superior-enla-era-de-la-neurocultura

Ruiz Fernández, M. (2014). Una experiencia de coaching en estudiantes universitarios International Journal of Developmental and Educational Psychology. Revista Infad de Psicología, 4(1).

Ruiz, M. I., Boada-Grau, J., Tejedor, E. M., y Ficapal-Cusí, P. (2016). Una experiencia de coaching en estudiantes universitarios. International Journal of Developmental and Educational Psychology. Revista INFAD de Psicología, 4(1), 515-518.

Sánchez Mirón, B., y Boronat Mundina, J. (2014). Coaching Educativo: Modelo para el desarrollo de competencias Intra e Interpersonales. Educación XX1, 17(1), 221-242.
Sánchez-Teruel, D. (2013). El coaching pedagógico dentro del sistema educativo: innovando procesos. Revista Intercontinental de Psicología y Educación, 15(2), 171191

Whitmore, J. (2011). Coaching, el método para mejorar el rendimiento de las personas. México DF: Paidós. 